\title{
Design and Implementation of Indoor Environment Parameter Monitoring System for Smart Home
}

\author{
Ling-jie Kong \\ Department of Physics and Electronic Engineering, Heze University, \\ Heze, Shandong 274015, China \\ kljie1000@163.com
}

\begin{abstract}
According to the reports shown by World Health Organization, the four factors that affect comfort of human living are environment temperature, humidity, light intensity and $\mathrm{CO}_{2}$ concentration in the air.

Therefore, in order to intelligently control and improve living environment in a real-time acquisition method, a kind of indoor environment parameter monitoring system for smart home is designed in this paper, which based on the Zigbee chip CC2530F256 microprocessor. Firstly, we choose CC2530F256 microprocessor as the main control chip of this system. Secondly, we select the DS18B20 as temperature sensor, the HM1500LF as humidity sensor, the CdS photosensitive resistance LXD3526 as photoelectric sensor and the MG811 as $\mathrm{CO}_{2}$ gas sensor.

After determining the device selection, we complete system hardware design by using Designer Altium 6.9, according to design philosophy of function modularization. This system hardware design mainly consists of five parts, including the CC2530F256 main control module, the sensor module, the liquid crystal display module, the sound and light alarm module, and the serial communication module. Then, this system software design is completed in integrated development environment that named Embedded Workbench for IAR 8051 V8.10 by using structured programming ideas. And the main function of this program is to complete tasks of collection, display, alarm and communication for indoor environment parameters. Afterwards, a test platform is set up, which can carry on testing for indoor environment parameter monitoring system. When testing for each functional module, the overall performance of this system is also done. Lastly, the test results show that this indoor environment parameters monitoring system can complete predetermined functions with high reliability and practical value, which providing a reference example for similar products in view of design and development point.
\end{abstract}

Keywords: Smart home; Zigbee; CC2530F256; indoor environment parameter monitoring system

\section{Introduction}

As everyone knows that Internet of Things is considered as another technological revolution following the PC and the Internet under development course of information industry. And as one of the main application areas of Internet of Things, smart home has already become the development trend in current society. The so-called smart home is a home automation system that integrated with various sub-systems such as intelligent building, communication networks, environment monitoring, entertainment video and household appliances lighting. That means that the people want to get a more comfortable, security, health and intelligent living or working environment in the house [1] [2]. It can be seen that the environment data acquisition system is an important part of smart home, which makes the collection and transmission of running information for various equipments as a key to ensure normal operation of the smart home system. Consequently, 
whether can we effectively achieve real-time monitoring of indoor environment parameters for smart home has already becoming an important issue that should been resolved.

For one thing, under comprehensive utilization of computer technology, communication technology, and measurement and control technology, the main purpose of this indoor environment parameter monitoring system is to realize collection, recording and display for all kinds of physical parameters, and even to change these analog signals into digital quantity. For another, the main purpose of this indoor environment parameter monitoring system is to give processed data and results back to the user or the control center. Briefly speaking, it is a good solution to construct the electronic monitoring device by using appropriate sensor technology [3].

As a result, this paper introduces a kind of indoor environment parameter monitoring system for smart home that based on Zigbee chip CC2530F256 microprocessor, and describes the selection of components, hardware design and software design of this system in detail. In order to realize system transplantation on Z-stack protocol stack, and complete wireless data acquisition and transmission of indoor environment parameters for smart home in real time by using Zigbee network technology, this research will ultimately establishing a solid and practical foundation for automatic monitoring the indoor environment parameters.

\section{Overall Design of System}

The core of this indoor environment parameter monitoring system for smart home is Zigbee chip CC2530F256 microprocessor, allocated with temperature sensor module, humidity sensor module, photoelectric sensor module, $\mathrm{CO}_{2}$ gas sensor module, LCD12864 display module, sound and light alarm module and serial communication conversion module [4]. The overall hardware design structure block diagram of indoor environment parameter monitoring system is shown in Figure 1.

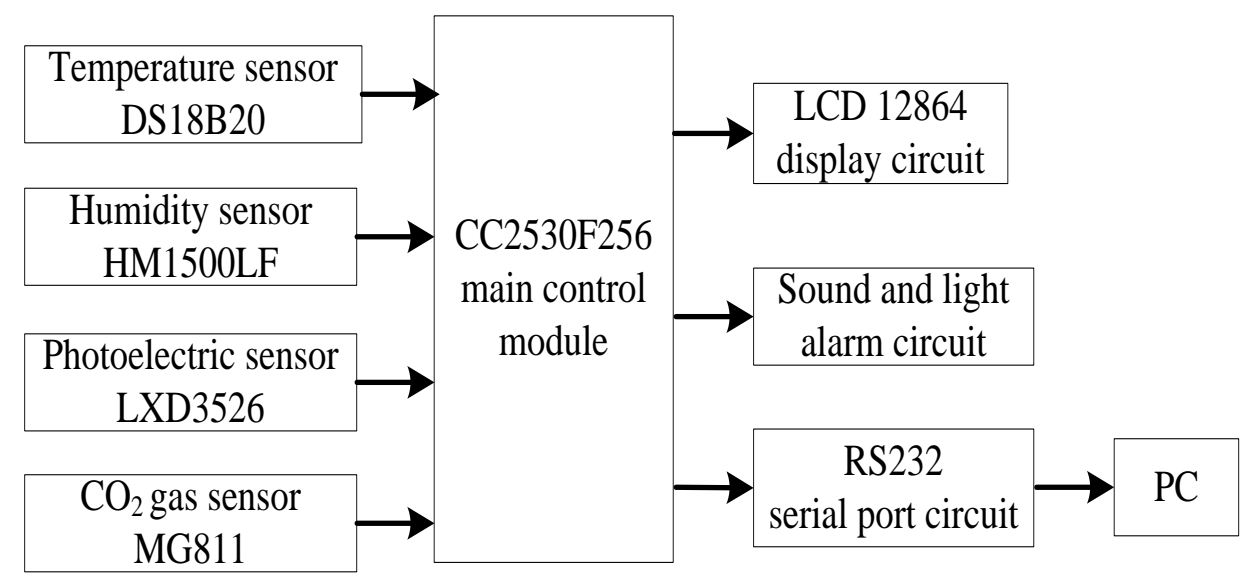

\section{Figure 1. Overall Hardware Design Structure Block Diagram of Indoor Environment Parameter Monitoring System}

The main function of this indoor environment parameter monitoring system is to convert non-electrical quantity of temperature, humidity, light intensity and $\mathrm{CO}_{2}$ concentration into electrical quantity output through temperature sensor, humidity sensor, photoelectric sensor and $\mathrm{CO}_{2}$ gas sensor. And after conversion processing of $\mathrm{CC} 2530 \mathrm{~F} 256$ microprocessor, the indoor environment parameter monitoring results will be displayed by LCD12864. Moreover, through RS232 serial conversion circuit, the indoor environment parameters are also been transmitted to upper PC computer for displaying. 


\section{Hardware Design of System}

\subsection{CC2530F256 Main Control Module}

The core chip of this indoor environment parameter monitoring system is CC2530F256 microprocessor which belongs to TI company. Meanwhile, it belongs to a CMOS SOC chip, embedded with high performance and low power consumption of enhanced 8051 microcontroller core, and integrated with 8 channel resolutions and adjustable 12 bit analog-to-digital conversion module and RF wireless transceiver (using $2.4 \mathrm{GHz}$ ) that conformed to IEEE 802.15.4 standard. So, the CC2530F256 microprocessor has excellent wireless receiver sensitivity and strong anti disturbance ability. At the same time, the CC2530F256 microprocessor can facilitate its own wireless communication network through Z-Stack protocol stack, which providing the users with a complete and powerful Zigbee solution [5] [6].

Among this indoor environment parameter monitoring system, such as four parts of the CC2530F256 minimum system control circuit, power circuit, JTAG interface circuit, CC2530F256 socket board and slot circuit can be referred as CC2530F256 main control module. And the hardware design circuit of CC2530F256 main control module is shown in Figure 2.

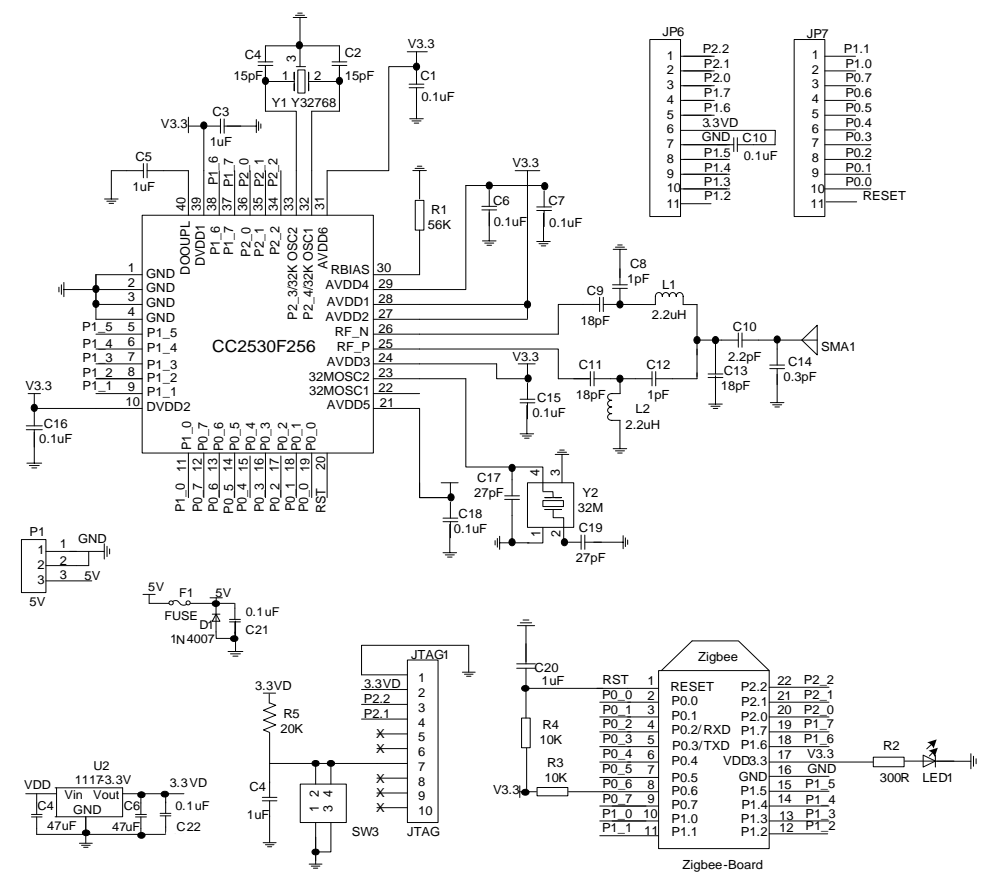

Figure 2. Hardware Design Circuit of CC2530F256 Main Control Module

\subsection{Sensor Module}

3.2.1. Temperature Sensor Module: This indoor environment parameter monitoring system uses single-bus intelligent and digital temperature sensor DS18B20 to accomplish collection of temperature information. It is a sensor that launched by United States DALLAS corporation. Its power supply voltage range is $3 \mathrm{~V}$ to $5.5 \mathrm{~V}$, and its temperature measurement range is $-55^{\circ} \mathrm{C}$ to $+125^{\circ} \mathrm{C}$ [7]. As a new type of intelligent and digital temperature sensor, the DS18B20 could directly output digital quantity under the help of internal integrated temperature sensor module, A/D conversion module and single data output control module, which making it easy to connect single chip or other microprocessors with simple interface circuit. Accordingly, the DS18B20 could be widely 
used in long distance, multi-node and distributed temperature detection field [8]. The hardware design circuit of temperature sensor module is shown in Figure 3.

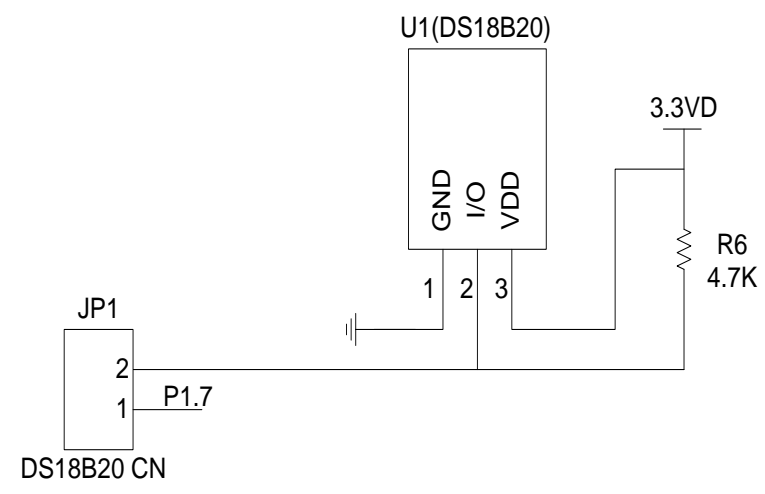

Figure 3. Hardware Design Circuit of Temperature Sensor Module

Seen from Figure 3, the DS18B20 can be connected to general I/O port P1.7 of CC2530F256 microprocessor by a single bus connection mode. Because the temperature sensor DS18B20 has unique product serial number, it will be easy to accomplish distributed multi-point temperature monitoring task in practical application.

3.2.2. Humidity Sensor Module: This indoor environment parameter monitoring system uses HM1500LF as its humidity sensor. It is a kind of linear voltage output and integrated humidity sensor, which belongs to French Humirel corporation. By using $5 \mathrm{~V}$ power supply with constant pressure, the HM1500LF can output a proportional relationship between voltage level signal and relative humidity. Moreover, the HM1500LF has many outstanding characteristics, such as built-in amplifying circuit, fast response speed, good repeatability and strong anti-interference ability [9]. The hardware design circuit of humidity sensor module is shown in Figure 4.

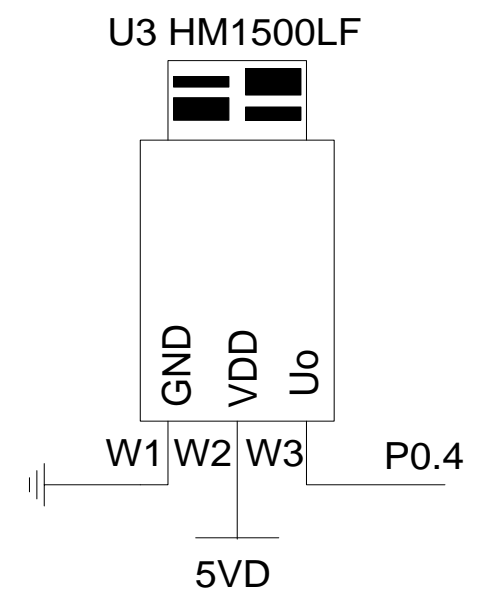

\section{Figure 4. Hardware Design Circuit of Humidity Sensor Module}

3.2.3. Photoelectric Sensor Module: This indoor environment parameter monitoring system employs CdS photoresistor LXD3526 as its photoelectric sensor. Its bright resistance (when the sun is in direct sunlight) is only $8 \mathrm{~K} \Omega$ to $20 \mathrm{~K} \Omega$, and its dark resistance (when there is no visible light) can be up to $0.5 \mathrm{M} \Omega$. When photoresistor LXD3526 is connected with $51 \mathrm{~K} \Omega$ resistor R7 in series way, the $3.3 \mathrm{~V}$ would be decomposed into two parts voltage. So, the voltage value obtained by both ends of photoresistor LXD3526 can be used to represent light intensity [10] [11]. The hardware design circuit of photoelectric sensor module is shown in Figure 5. 


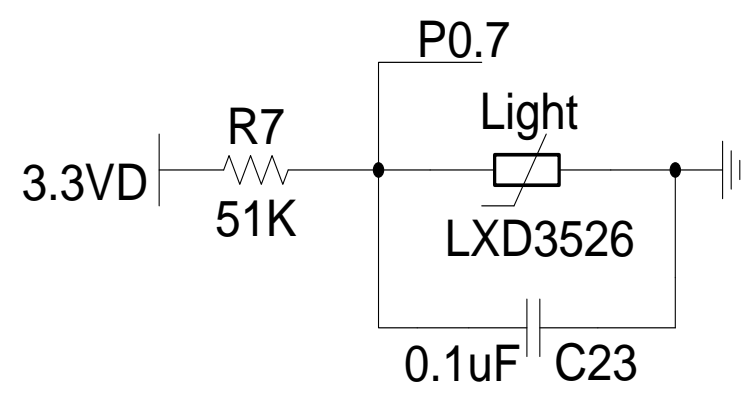

Figure 5. Hardware Design Circuit of Photoelectric Sensor Module

3.2.4. $\mathrm{CO}_{2}$ Gas Sensor Module: This indoor environment parameter monitoring system adopts MG811 as its $\mathrm{CO}_{2}$ gas sensor. When $\mathrm{CO}_{2}$ concentration in the air is higher, the output voltage of MG811 is lower. And by contrary, when $\mathrm{CO}_{2}$ concentration in the air is lower, the output voltage of MG811 is higher [12]. And the range of $\mathrm{CO}_{2}$ concentration in the air is generally $300 \mathrm{ppm}$ to $1500 \mathrm{ppm}$, which corresponding with output voltage for $325 \mathrm{mV}$ to $300 \mathrm{mV}$. Therefore, in order to enter into CC2530F256 microprocessor for sampling, the MG811 output voltage will be amplified through CA3140 for 3 times. The hardware design circuit of $\mathrm{CO}_{2}$ gas sensor module is shown in Figure 6.

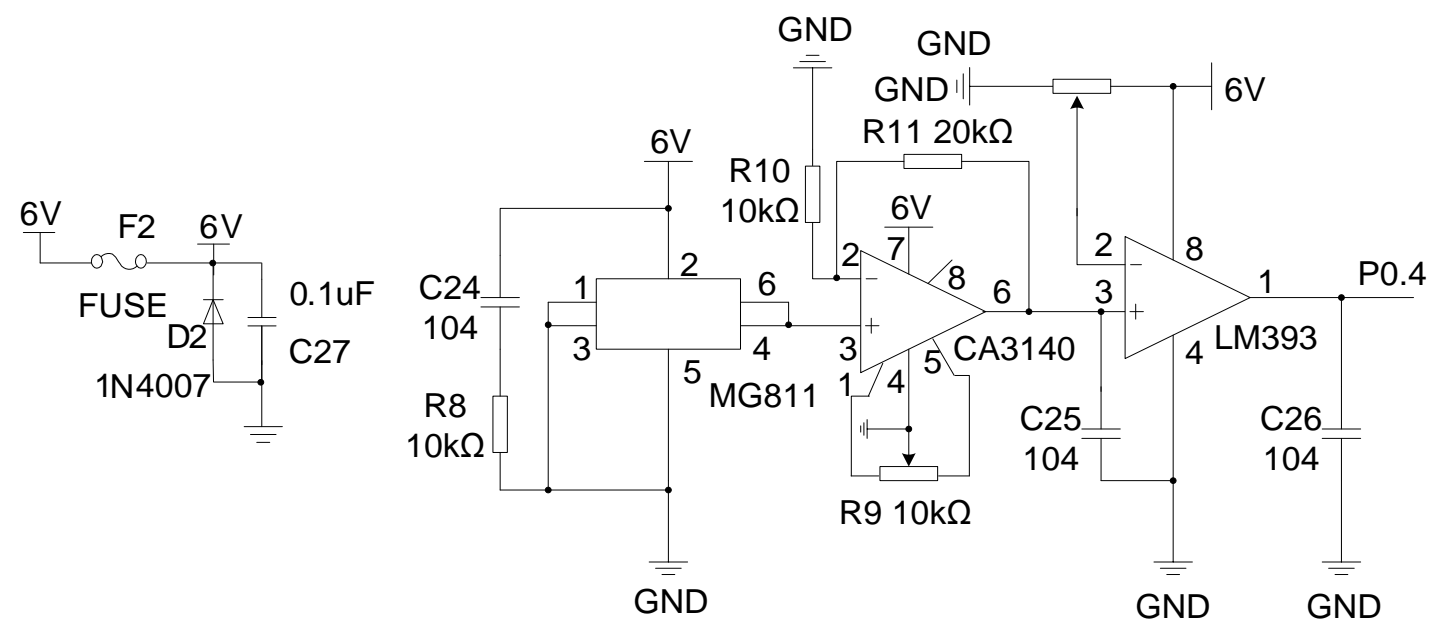

Figure 6. Hardware Design Circuit of $\mathrm{CO}_{2}$ Gas Sensor Module

Seen from Figure 6, the supply voltage of MG811 is 6V, which can be supplied by $220 \mathrm{~V}$ to $6 \mathrm{~V}$ power adapter.

\subsection{Liquid Crystal Display Module}

This indoor environment parameter monitoring system chooses LCD12864 monochrome screen as its liquid crystal display module. It is connected with parallel interface of CC2530F256 microprocessor, so that the SPI bus is indirectly controlled to complete driving work. The hardware design circuit of Liquid Crystal Display module is shown in Figure 7. 


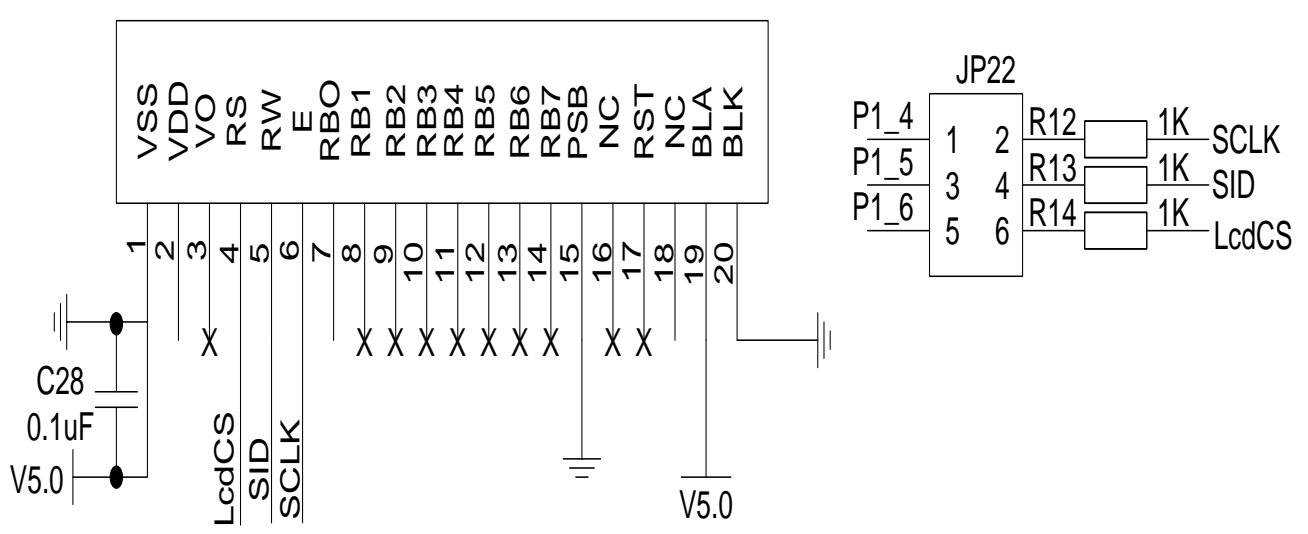

Figure 7. Hardware Design Circuit of Liquid Crystal Display Module

\subsection{Sound and Light Alarm Module}

This indoor environment parameter monitoring system selects a piezoelectric passive buzzer as its sound alarm module. When the temperature, humidity, light intensity and $\mathrm{CO}_{2}$ concentration are less than preset upper limit value or greater than preset lower limit value, we can control general I/O port P2.0 of CC2530F256 microprocessor to output a high level, so that the buzzer does not work. On the contrary, when the temperature, humidity, light intensity and $\mathrm{CO}_{2}$ concentration are greater than preset upper limit value or less than preset lower limit value, we can control general I/O port P2.0 of CC2530F256 microprocessor to output a low level, so that the buzzer can achieve automatic sound alarm function for indoor environment parameter information. Besides, in order to accurately identify sound alarm that corresponding with different types of indoor environment parameters, this indoor environment parameter monitoring system also uses LED as a flashlight alarm module. And it is a solid state semiconductor device which can directly convert electrical energy into light energy. The hardware design circuit of sound and light alarm module is shown in Figure 8.
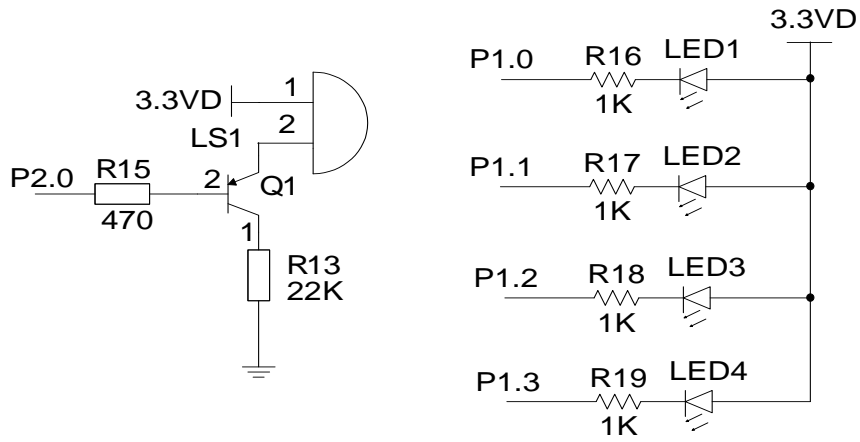

Figure 8. Hardware Design Circuit of Sound and Light Alarm Module

\subsection{Serial Communication Module}

In order to realize transparent data communication between indoor environment parameter monitoring system and upper PC computer, the RS232 serial port circuit is also designed. Serial communication mode is a more commonly used communication method, which only needs two lines (that is TXD and RXD) to achieve full duplex communication among two digital devices. Between UART of CC2530F256 microprocessor and RS232 interface of upper PC computer, there is a serial converter achieved by MAX232. It belongs to Maxim corporation. In this way, the upper PC computer can directly receive and display four parameters named temperature, humidity, light intensity and $\mathrm{CO}_{2}$ 
concentration by using serial port tool. The hardware design circuit of serial communication mode is shown in Figure 9.

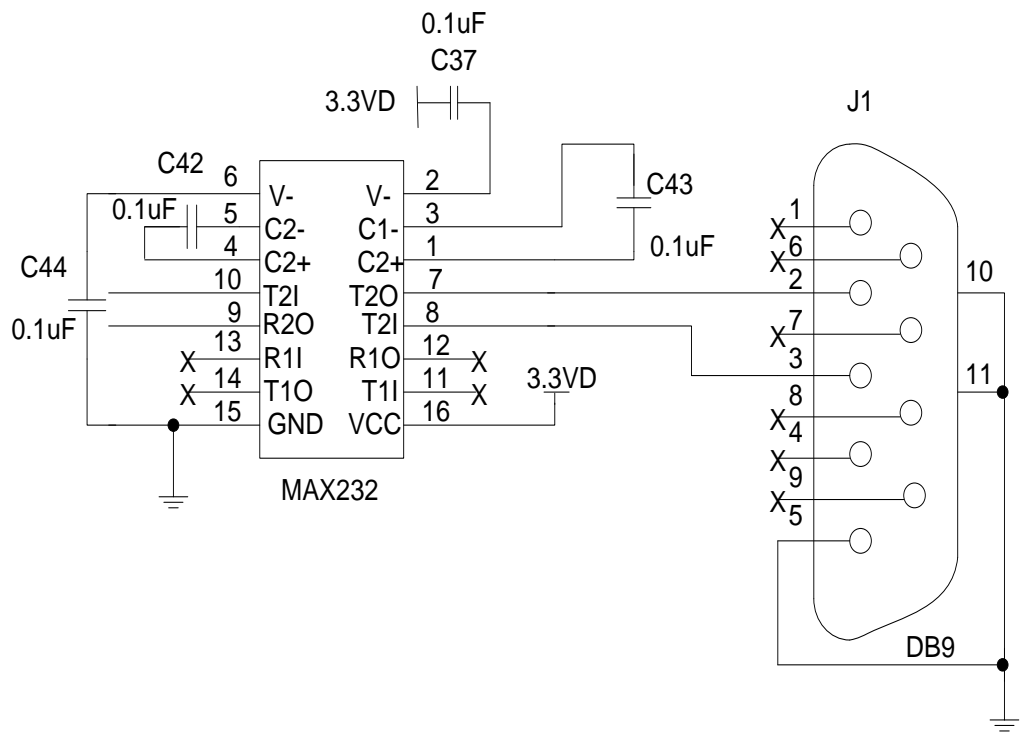

\section{Figure 9. Hardware Design Circuit of Serial Communication Module}

\section{Software Design of System}

System software design is written by $\mathrm{C}$ language that based on integrated development environment of Embedded Workbench for IAR 8051 V8.10. And the modular design concept is adopted to facilitate project management and modification.

The main idea of this system software design is mainly depended on query method. Firstly, we divide this system into some modules according to its function. Secondly, we compile relevant subroutine according to these modules. Lastly, the concrete method of this system software design and implementation is to call system self-checking subroutine, indoor environment parameter monitoring subroutine and serial communication subroutine through main program.

\subsection{System Self-checking Subroutine}

The function of this system self-checking subroutine is to complete function test for these four types of sensors, so as to test whether these four types of sensors are working properly or not, and select an appropriate data weight in system working environment.

After power on, this system firstly call system self-test function of system_check ( ) to carry out self inspection. Prior to self inspection, the program will go through a delay about $500 \mu$ s to prevent this system from reading error caused by instability when system starting. And after self inspection, the program will start A/D converter in turn and read the corresponding sensor data for these four types of sensors.

As can be seen in Figure 4, Figure 5, Figure 6 and Figure 7, if the temperature sensor or humidity sensor or photoelectric sensor appears malfunction, even it is pulled out. The input voltage of the A/D converter for $\mathrm{CC} 2530 \mathrm{~F} 256$ microprocessor will be set as $3.3 \mathrm{~V}$, and its corresponding conversion result is 8192 . Then this data will be used as a criterion for judging whether these sensors are working correctly or not. And if the $\mathrm{CO}_{2}$ gas sensor fails or it is pulled out, the input voltage of the A/D converter for CC2530F256 microprocessor will be set as $0 \mathrm{~V}$, and its corresponding conversion result is 0 . In the same way, this data will be used as a criterion for judging whether $\mathrm{CO}_{2}$ gas sensor is working correctly or not. However, in the process of self inspection, the error messages of these sensors will be displayed in a timely manner. At the same time, it is necessary to hang-up 
the system to avoid causing damages.

After four types of sensors can be confirmed to work properly by system self-checking subroutine, then the "SYSTEM IS NORMAL" information will be displayed in LCD12864 screen. And, the system self-checking subroutine could carry out weight allocation for sensor data according to weight distribution table. Finally, the system enters into a normal operation state. The flow chart of system software design for self-checking subroutine is shown in Figure 10.

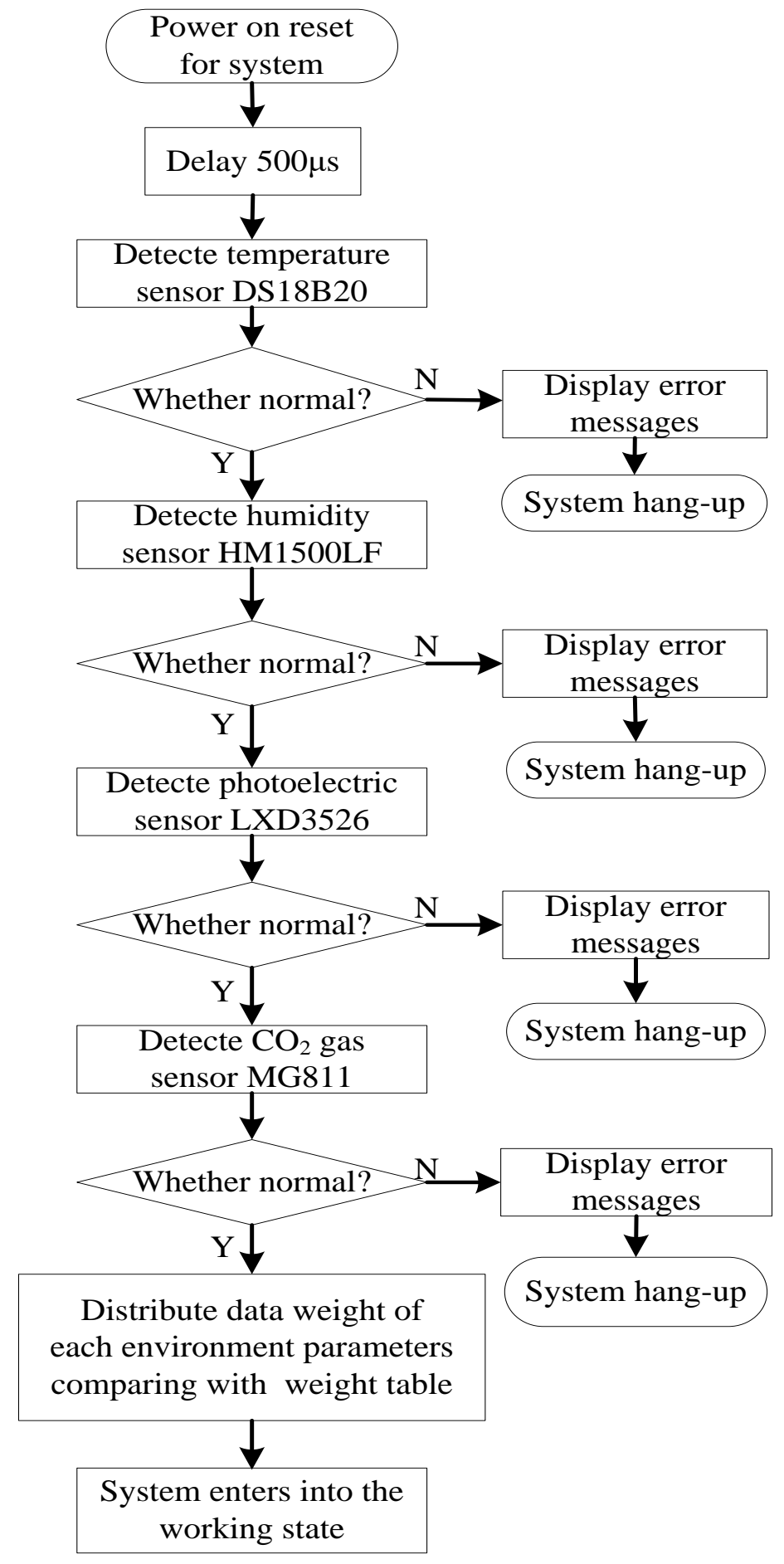

Figure 10. The Flow Chart of System Software Design for System Self-checking Subroutine 


\subsection{Indoor Environment Parameter Monitoring Subroutine}

The main function of indoor environment parameter monitoring subroutine is to achieve sensor data reception, data analysis and judgment, driving LCD12864 to display and carrying out sound and light alarm. If this system is operating normally, then this system will enter the loop to detect monitor state of the data returned by each sensor. After returning information by each traverse of these sensors, we carry out data analysis and determine whether these sensors occur abnormal situation or not, according to the weight algorithm design. When abnormal condition happened, the corresponding message prompt, sound and light alarm prompt will be started. And the flow chart of system software design for indoor environment parameter monitoring subroutine is shown in Figure 11.

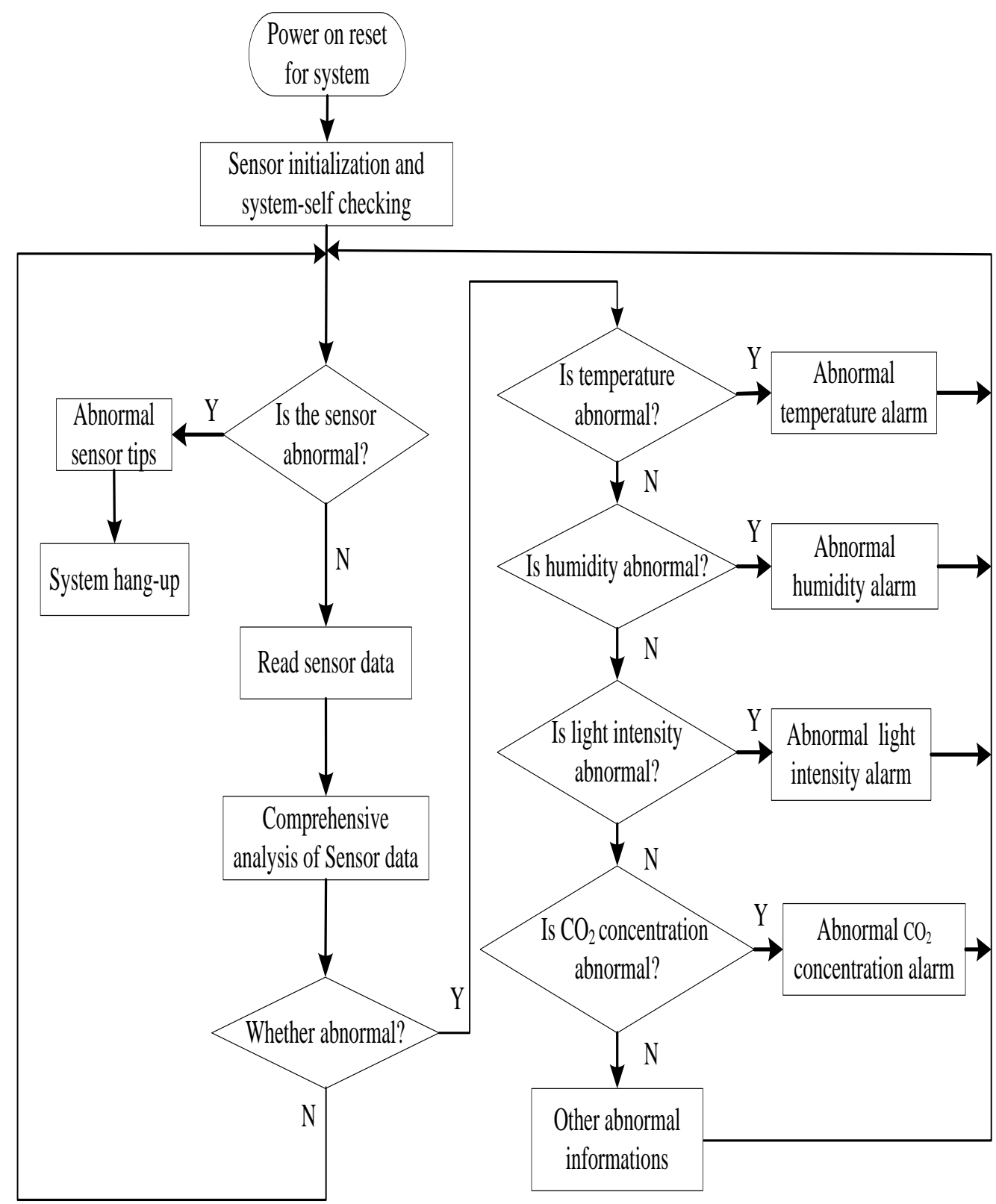

Figure 11. The Flow Chart of System Software Design for Indoor Environment Parameter Monitoring Subroutine 


\subsection{Serial Communication Subroutine}

The main function of serial communication subroutine is to complete transparent data communication between the indoor environment parameter monitoring system and the upper PC computer. And the flow chart of system software design for serial communication subroutine is shown in Figure 12.

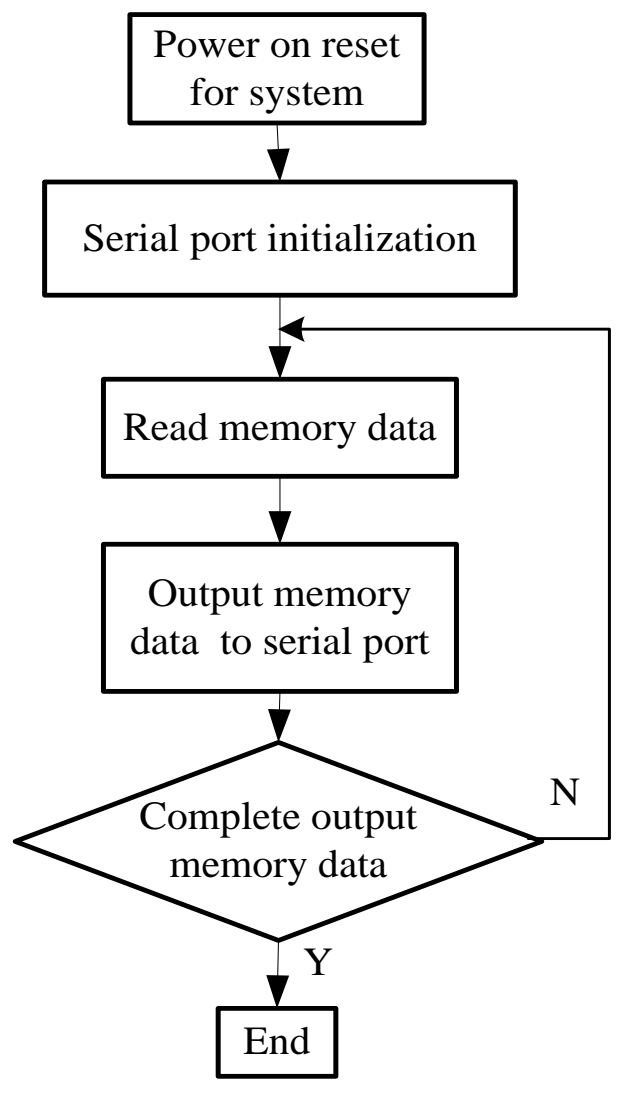

\section{Figure 12. The Flow Chart of System Software Design for Serial Communication Subroutine}

\section{System Test}

In order to test the performance of this indoor environment parameter monitoring system, we establish an actual circuit according to the above system hardware and software design. Also, we complete these four kinds of parameters acquisition for indoor temperature, humidity, light intensity and $\mathrm{CO}_{2}$ concentration under simulated smart home. Among them, the indoor temperature standard value of simulated smart home is 12 degrees Celsius, the indoor humidity standard value is $25 \% \mathrm{RH}$, the indoor light intensity standard value is $1300 \mathrm{LX}$ and the indoor $\mathrm{CO}_{2}$ concentration standard value is $450 \mathrm{ppm}$. Then the system test results are shown in Table 1. 
Table 1. System Test Results

$\begin{array}{ccccc}\text { No. } & \begin{array}{c}\text { Temperature } \\ \left({ }^{\circ} \mathrm{C}\right)\end{array} & \begin{array}{c}\text { Humidity } \\ (\mathrm{RH})\end{array} & \begin{array}{c}\text { Light Intensity } \\ (\mathrm{LX})\end{array} & \begin{array}{c}\mathrm{CO}_{2} \text { Concentration } \\ (\mathrm{ppm})\end{array} \\ 1 & 12.6 & 28 & 1362 & 483 \\ 2 & 12.9 & 31 & 1421 & 472 \\ 3 & 13.2 & 33 & 1513 & 480 \\ 4 & 12.8 & 29 & 1382 & 476 \\ 5 & 13.4 & 36 & 1552 & 481\end{array}$

As can be seen from Table 1, this indoor environment parameter monitoring system has a high measuring accuracy and obtains satisfactory results.

\section{Conclusion}

As a kind of intelligent equipment aiming at collection and transmission of environment data, this indoor environment parameter monitoring system plays a very important role in smart home. Therefore, combining with two advantages of digital communication and network function for modern smart home system, this paper introduces a complete set of design scheme for indoor environment parameter monitoring system based on Zigbee chip CC2530F256 microprocessor.

The CC2530F256 microprocessor not only improves the anti-interference ability of this system, but also ensures the long-term stability. At the same time, these four types of sensor modules used in this design scheme not only simplify the hardware design, but also improve the measurement accuracy, so that the whole system can be easily operated and maintained. After field debugging various basic functions of indoor environment parameter monitoring system in simulated smart home, the testing results show that this system can achieve predetermined functional requirements with high reliability and practical value.

Although, this indoor environment parameter monitoring system for smart home can preferably get exact value of temperature, humidity, light intensity and $\mathrm{CO}_{2}$ concentration. However, this system is still not perfect. It means that this system should need some further improvements. For example, this system can only get a small range of environment parameters information, and there is no wireless communication part.

\section{References}

[1] P. Yong and L. Guangming, "Study on smart home system based on ZigBee", Computer Engineering \& Design, vol. 35, no. 5, (2014), pp. 1547-1550.

[2] X. Hailiang, Z. Peisi, Z. Shaoqi and Y. Yingjing, "Research of smart home furnishing control system by internet of things based on ZigBee", Application of Electronic Technique, vol. 39, no. 12, (2013), pp. 79-81.

[3] W. Yi and B. Chunlan, "Design of smart home system based on GSM and ZigBee technology", Journal of Hebei University of Technology, vol. 43, no. 1, (2014), pp. 15-18.

[4] M. Kun, "Design of MSP430 based smart home system", Suzhou University, (2014), pp. 35-38.

[5] Texas Instruments, A True System-on-Chip Solution for 2.4-GHz IEEE 802.15.4 and ZigBee Applications. http://www.ti.com.cn/cn/lit/ds/symlink/cc2530.pdf, 2010-10-05.

[6] Y. Jiting, Y. Jia, J. Zhiman, W. Bin, Z. Zaifang and Y. Jianbo, "Research and development of smart home control system based on ARM and ZigBee", Computer Measurement \& Control, vol. 21, no. 9, (2013), pp. 2451-2454.

[7] Z. Yuning and Z. Ying, "Digital temperature measuring devices based on DS18B20", Mechanical Engineering \& Automation, vol. 41, no. 4, (2012), pp. 149-151.

[8] L. W. Xi, H. Xiao, T. Dong, Z. Hui and J. Cuoping, "Design of wireless temperature sensor network based on ZigBee technology", Transducer \& Microsystem Technologies, vol. 28, no. 4, (2009), pp. 69-71.

[9] Y. Shunming, Z. Hailan and Y. Ran, "SCM-based temperature and humidity control system for vegetable greenhouse", Modern Electronics Technique, vol. 34, no. 7, (2011), pp. 129-131.

[10] Z. Pinlin and X. Ping, "Greenhouse temperature and light control system based on MSP430 MCU", Popular Science \& Technology, vol. 11, no. 8, (2009), pp. 149-150. 
[11] H. Chao, "The samrt home of data acquisition and control system based on ZigBee technology", Taiyuan University of Technology, (2011), pp. 30-32.

[12] D. Hao, "Design and implementation of a system to locate elderly living alone and to monitor indoor environment", Huazhong Normal University, (2013), pp. 28-31.

\section{Author}

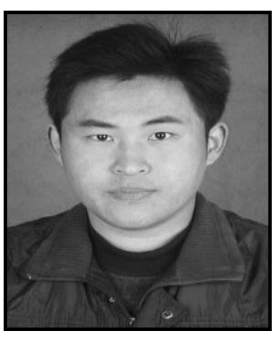

Ling-jie Kong, He received his Master degree in detection technology and automatic equipment from Lanzhou University of Technology in China. Now he is an assistant in Heze University of Shandong province. His major research direction is detection technique and automatic device. 